\title{
Aflatoxicosis in a white pekin duck
}

\author{
K.J. Bibu \\ Kerala Agricultural University Poultry and Duck Farm, Kerala, India. \\ E-mail : bibujohn@rediffmail.com \\ Published online at www. veterinaryworld.org on 25-03-2011
}

\section{Introduction}

Aflatoxicosis represents one of the serious diseases of poultry, livestock and other animals. The cause of this disease in poultry and other foodproducing animals has been attributed to the ingestion of various feeds contaminated with Aspergillus flavus. This toxigenic fungus is known to produce a group of extremely toxic metabolites, of which aflatoxin $\mathrm{B}_{1}$ $\left(\mathrm{AFB}_{1}\right)$ is most potent. Avian species especially chickens; goslings, ducklings and turkey poults are most susceptible to $\mathrm{AFB}_{1}$ toxicity. The incidence of hepatocellular tumors, particularly in ducklings, is considered to be one of the serious consequences of aflatoxicosis (Dalvi,1986). This article reports a case of aflatoxicosis in a 15 month old white pekin duck.

\section{Case history and Observations}

A 15 month old white pekin duck was found to be dead in a flock of 80 birds reared in the University Poultry and Duck Farm, Mannuthy, Thrissur, Kerala during the month of February 2010. The flock was showing reduction in feed intake for the last two weeks but there the egg production did not show a much variation. External examination of carcass did not show any disease changes.

On post mortem examination, the carcass revealed large cirrhotic pale friable liver with perihepatitis. Heart, Lungs, Spleen and kidney were normal. Salphingitis, impaction of the oviduct with cheesy material and egg peritonitis were also noted. The case was diagnosed as aflatoxicosis.

\section{Discussion}

Case reports of aflatoxicosis in ducks are rare. Chronic exposure to low levels of aflatoxin can result in cancer and immunosuppression (Sharma, 1993). This immunosuppression has caused other bacteria to flare up causing egg peritonitis, impaction of the oviduct and salphingitis. Decreased feed intake along with increased liver weights in birds is consistent with earlier reports (Verma et al., 2004; Ortatatli and Oguz, 2001). Even though prevention and avoidance are the best way to control aflatoxicosis, natural contamination of crops with A. flavus is sometimes unavoidable. Such aflatoxin-contaminated feeds may have caused the death and reduction in feed intake. The feeds can be decontaminated using various methods which mainly focus on physical removal or chemical inactivation of the toxins in the feeds. Moreover, dietary additives such as activated charcoal, phenobarbital, cysteine, glutathione, betacarotene, fisetin and selenium have also been reported to be effective in the reduction of aflatoxicosis in poultry (Dalvi, 1986).

\section{References}

1. Dalvi, R.R. (1986). An overview of aflatoxicosis of poultry: Its characteristics, prevention and reduction. Vet. Res. Commun. 10 (1):429-443.

2. Ortatatli, M., and H. Oğuz. (2001). Ameliorative effects of dietary clinoptilolite on pathological changes in broiler chickens during aflatoxicosis. Res. Vet. Sci.71:59-66.

3. Sharma, R. P. (1993). Immunotoxicity of Mycotoxins $J$. Dairy Sci. 892-897.

4. Verma, J., et.al.(2004). Effect of graded levels of aflatoxin, ochratoxin and their combinations on the performance and immune response of broilers. Br. Poultry Sci. 45:512-518. 